\title{
Fracture immobilization using round stainless wire
}

Nduka A.I.

Mawenzi Hospital, Kilimanjaro, Tanzania

Nduka A.I.: Fracture immobilization using round stainless wire. Tanz Dent J 2009; 15(2):45-47

Correspondence: Nduka A.I., Mawenzi Hospital, Kilimanjaro, Tanzania.

There are many different methods for fixation of the bony fragments of the facial-oral area. The best one depends on several factors, such as the extent of the fracture, number of remaining teeth, soft tissue damage, preferences and experience of the surgeon. Fractures of mandible and maxilla are commonly treated by either conservative or operative methods. A conservative method is used when the fracture is not complicated, not displaced, can easily be reduced and patient has good, strong and enough number of teeth. On the other hand, when the fracture is complicated, displaced and no enough teeth remaining, an operative approach becomes inevitable.

\section{Conservative method of fracture immobilization} The conservative method is divided into; first temporary or transportation method of immobilization e.g. parietal-submental bandage, interdental wiring and second; permanent method of immobilization e.g. eyelet method, plain stainless wire, standard arch bar, acrylic splints etc.

Round stainless wire with the diameter of $0.8 \mathrm{~mm}$ is the appropriate splint for maxilla/mandible fracture immobilization. The wire can be bent to conform well to the contours of dental arch lying on the neck of each tooth. It is applied only when there are two or more strong teeth on both fragments.

Flexible stainless wire splint is used as; plain wire splint clamp, bend plain wire splint conforming to a dental arch defect and inter-jaw plain wire splint with hooked loops.

General requirement for preparation and application of round stainless wire splints:

The general requirement for preparation and application of round stainless wire splints are; stainless wire of around $0.8 \mathrm{~mm}$ in diameter, fixing stainless wire of $0.4 \mathrm{~mm}$ diameter (Kirschin wire), crampon forceps (for bending stainless steel wire), needle holding or hemostat forceps, a pair of wire cutter scissors and a file, (Figure 1).

When preparing stainless wire splint, make sure that it fits well lying between the margin of the gum and equator of each tooth, conforming to contour of dental arch. Splint should never lie over the gum otherwise compression ulcer will occur on the gum. Splint should be firmly fixed to each tooth using stainless wire (Kirschin wire) with diameter 0.4 $\mathrm{mm}$. This always distributes force equally to each tooth making them one block.

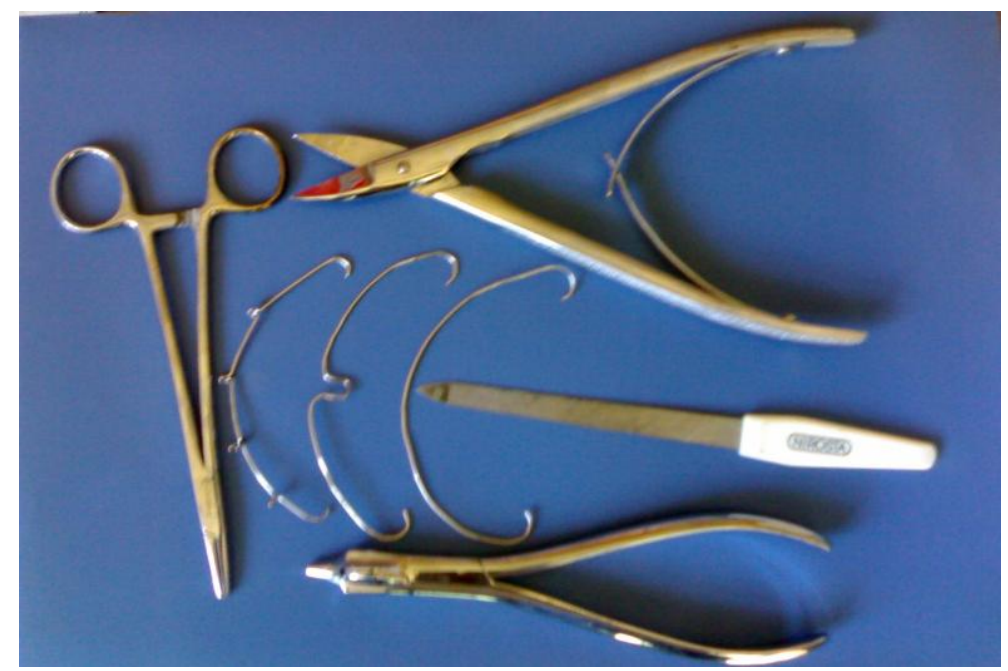

Figure 1: Requirement for preparation and application of round stainless wire splints 
General advantages of stainless wire splints:

The advantages of stainless wire splints are;

1. It is flexible so it can be bent easily to conform to contour of dental arch.

2. It can easily be firmly fixed to teeth using Kirschin wire.

3. Due to small surface area lying on the teeth, retention of food remnants is minimal and hence it is easy to maintain good oral hygiene.

4. Force is distributed to all teeth involved, especially when inter-jaws traction is used.

5. Is strong/firm enough to be used in one jaw when the fracture is not displaced or can be reduced easily

6. It can also be used during fracture of alveolar part of jaws.

7. Can be used with minimum number of strong teeth (2-3) on each fragment.

8. When the minimum period of immobilization is reached, inter jaw traction can be removed, leaving the splint on the fracture jaw for sometimes to stabilize the coleus formed.

9. Does not cause trauma to soft tissue when applied properly.

10. Can be used to immobilize luxated/ subluxated teeth.

11. Can be used when teeth are not strong enough (teeth with slight parodontose).

\section{Plain stainless splint - Clamp:}

In case there is no displacement-when the upper and lower teeth oppose one another in such a way that the bites are normal, plain stainless splint is used alone, without inter-maxilla fixation, as the splint is strong enough to hold the fragments in place as long as the patient takes soft food during the whole period of treatment.

Is used when, to the large fragment there is more than 3 strong teeth and to the small fragment not less than 2 strong teeth.

\section{Indications of plain stainless splint-clamp:}

1. Linear fracture to the mandible without displacement.

2. Fracture to mandible which can easily be reduced.

3. A fracture to the middle or lateral part of maxilla or mandibular alveolar.

4. Luxation injury to the teeth.

Bend stainless wire splint following defect on the teeth row

It is a variant of plain wire splint; it differs only when it is bent to follow the contour of defect on the teeth row. Rules for bending the splint are the same as those for the plain wire splint. On the defect where teeth are missing, bending should correspond to the width of lateral surface, or be not less than $2 / 3$ of the tooth the splint is laying on. The splint at the defect should lay on the alveolar ridge or not more than $5 \mathrm{~mm}$ above it.

This type of splint can be prepared even when using stainless wire splint with hooked loops if there is a defect in dental arch.

Indications for using a bend stainless wire splint:

1. Fracture mandible within teeth row limited without displacement.

2. Fracture which can easily be reduced.

3. When fracture line passes through part of the alveolar ridge with missing teeth.

4. Fracture mandible within teeth raw limits with bone tissue defect.

5. Can be applied in interjaws immobilization with hooked loop.

\section{Stainless wire splint with hooked loops}

Indications of stainless wire splint with hooked loops:

1. Fracture of mandible at the teeth row limits with displacement of fragment.

2. Fracture mandible with tight reduction of fragments.

3. Multiple fractures to the mandible.

4. Fracture mandible beyond teeth rows.

5. Fracture maxilla.

6. Fracture to both maxilla and mandible (there must be added a parietal- sub mental bandage).

Stainless wire splint with hooked loop is commonly used in inter-jaws immobilization. To each splint 56 hooked loops are made at equal intervals. To the large fragment 3-4 hook is 3-5 mm otherwise short hooks will not hold rubber bands and long hooks will cause trauma to the mucous membrane on the lips. On the maxilla hooks are directed upward and on the mandible hooks are directed downwards at angle of 35- 45 from the tooth axis. The apex of hooks must stand away about $2 \mathrm{~mm}$ from the alveolar mucous membrane to avoid likelihood of forming sores from rubber band pressure.

When preparing stainless wire splint with hooks, through anatomical orientation, at list hooks should lay along axis of second incisors, first premolar, and first molar. They should lay on the hard surface of teeth, not along inter-dental space. Make sure that both splints are firmly secured to the maximum number of teeth with wire ligatures so that after putting rubber bands the force is distributed to all teeth. 


\section{Reference:}

1. Naumann H. H. Head and Neck Surgery Volume 2. Face and Neck Skull. First edition. Geoge Tieme Publishers Stuttgart. 1980; Page 43-83.

2. Korte R. Primary Traumatology Part 2: experimental edition. German Agency for Technical Cooperation. 1982; Page 126-33.
Bernadsky U. I. Traumatology and Reconstructive Surgery-Maxillofacial Area. 3rd edition. Page 3666. Medical Literature. Moscow.1990

3. Robustova T. G. Stomatology 3rd edition. Page 268- 366. Medicine Moscow Surgery. 2003

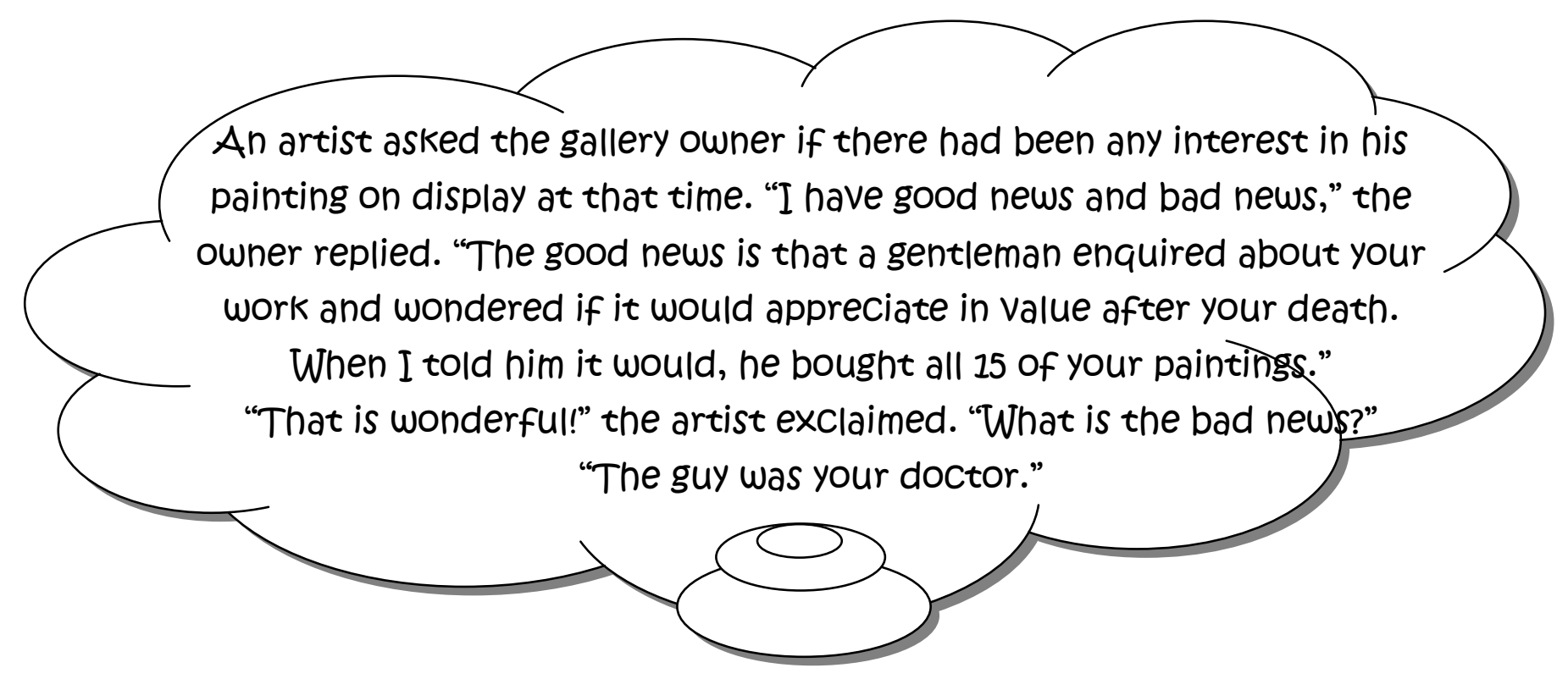

On an application form, Que is asked

to write against name, age,

qualification etc. He replied against

sex as "sometimes till get married." 